\title{
Hérnia diafragmática congênita com manifestação tardia: relato de caso
}

\section{Congenital diaphragmatic hernia with late manifestation: case report}

\author{
Alexsandra Ferreira da Costa Coelho, ${ }^{1}$ Glidiane Silva do Nascimento ${ }^{1}$ e Ana Rita Marinho Ribeiro Carvalho ${ }^{1}$ \\ ${ }^{1}$ Centro Universitário Maurício de Nassau
}

\section{Palavras-chave:}

Hérnia diafragmática; Dispneia; Anormalidades congênitas.

\section{Resumo}

Introdução: a hérnia diafragmática congênita é caracterizada pela formação incompleta do diafragma e sua incidência neonatal é cerca de 1:3.000 a 1:5.000 nascidos vivos. Cerca de 90\% dos pacientes apresentam disfunção respiratória nas primeiras 24 horas de vida. Objetivo: enfatizar a importância do diagnóstico precoce, estabelecido primordialmente durante o pré-natal, para uma conduta terapêutica adequada e demonstrar a importância dos exames de imagem para o diagnóstico em casos de manifestação tardia. Descrição do caso: lactente de 5 meses deu entrada em uma emergência localizada na zona agreste de Pernambuco, apresentando dispneia súbita, irritabilidade e gemência. A radiografia de tórax demonstrou dilatação do brônquio fonte esquerdo, que sugeriu aspiração de corpo estranho. 0 lactente foi transferido para um hospital de referência para realização de broncoscopia, sendo admitido com estado geral grave, importante desconforto respiratório e queda da saturação de oxigênio. A equipe médica solicitou de urgência uma tomografia de tórax cujo resultado foi sugestivo de hérnia diafragmática. Em seguida, o lactente foi submetido à videotoracoscopia para correção do defeito congênito, através da herniorrafia diafragmática. O lactente evoluiu com evidente melhora do desconforto respiratório após o procedimento, recebendo alta hospitalar no terceiro dia do pós-operatório. Discussão: a hérnia diafragmática congênita com manifestação tardia pode ter apresentação clínica variável o que dificulta o seu pronto-diagnóstico e aumenta as chances de complicações. Para o diagnóstico diferencial, os exames de imagem tornam-se importantes, sobretudo a radiografia contrastada e a tomografia computadorizada de tórax, pois complementam o estudo de uma condição menos comum.

\section{Keywords:}

Hernia, diaphragmatic; Dyspnea; Congenital abnormalities.

\begin{abstract}
Introduction: congenital diaphragmatic herniation is characterized by incomplete diaphragm formation and neonatal incidence is about 1:3,000 to 1:5,000 liveborns. Almost ninety percent of the patients present respiratory dysfunction in the first twenty-four hours of life. Objective: to emphasize the importance of early diagnosis, established primarily during prenatal care, for the appropriate therapeutic management. Case description: a five-month-old infant was admitted to the emergency of a hospital located in the Agreste region of Pernambuco with sudden dyspnea, irritability, and moans; chest radiography demonstrated left bronchial dilation, which suggested foreign body aspiration. The child was transferred to a Reference Hospital to perform bronchoscopy, being admitted with a severe general condition, significant respiratory discomfort and a drop in oxygen saturation. The medical team urgently requested a chest tomography, which suggested diaphragmatic herniation. The patient was then submitted to videothoracoscopy for the surgical correction of congenital malformation. The patient had an evident improvement in respiratory discomfort after the procedure, being discharged on the third postoperative day. Discussion: congenital diaphragmatic herniation with late manifestation may have a variable clinical presentation, which hinders its prompt diagnosis and increases the chances of complications. For differential diagnosis, imaging tests become important, especially contrast radiography and chest tomography, since they complement the study of a less common condition.
\end{abstract}




\section{INTRODUÇÃO}

A hérnia diafragmática congênita (HDC) define-se como a ausência do desenvolvimento de parte ou da totalidade de uma hemicúpula diafragmática que ocorre em torno da nona semana de gestação, com herniação de vísceras abdominais para o interior do tórax. Constitui $8 \%$ das malformações congênitas e estima-se que a incidência da hérnia diafragmática congênita seja de um caso para cada 2 mil a 5 mil nascimentos. ${ }^{1}$

A maioria dos pacientes apresenta sinais e sintomas logo após o nascimento, como taquipneia, tiragens supraclavicular e intercostal, cianose, abdome escavado, assimetria torácica. Além disso, o murmúrio vesicular pode não ser audível no lado acometido, o que pode, ocasionalmente, levar ao diagnóstico errôneo de pneumotórax hipertensivo, acompanhado por distensão abdominal causada pelo rebaixamento do diafragma. ${ }^{2}$ Cerca de 5 a $10 \%$ podem manifestar essa anomalia congênita tardiamente ou, mesmo, nunca expressá-la. ${ }^{3}$ Apesar de menos frequente, a manifestação tardia da HDC é bem variada, o que dificulta o pronto-diagnóstico dessa patologia e aumenta as chances de o paciente apresentar complicações potencias: encarceramento e estrangulamento de vísceras herniadas, insuficiência cardiorrespiratória entre outras. ${ }^{4}$

A HDC pode ser diagnosticada no período pré-natal por ecografia em cerca de $60-80 \%$ dos casos, e há referência do seu diagnóstico pré-natal por ecografia já às 15 semanas de gestação. Tal fato é muito importante, uma vez que permite um tratamento precoce do neonato antes mesmo que este venha a apresentar sintomas. Quando a manifestação dessa anomalia congênita é tardia, a radiografia de tórax e a tomografia computadorizada tornam-se importantes, pois permitem o diagnóstico diferencial de uma condição menos comum com uma clínica variada que, se não diagnosticada precocemente, pode complicar com sérios riscos de vida. ${ }^{5,6}$

Os principais objetivos são destacar a importância do diagnóstico precoce e a necessidade do auxílio dos exames de imagens para a confirmação dos casos suspeitos de HDC, com o intuito de diminuir os riscos de complicações fatais advindas dessa patologia.

\section{DESCRIÇÃO DO CASO}

Lactente de 5 meses e 23 dias, sexo masculino, deu entrada num pronto-socorro no interior de Pernambuco apresentando dispneia súbita, irritabilidade e gemência. Genitora negava febre, tosse ou engasgo; a radiografia de tórax (Figura 1) demonstrou dilatação do brônquio fonte esquerdo. O lactente foi transferido para um grande hospital em Recife para realização de broncoscopia. O lactente foi admitido com estado geral grave, taquidispneico $(3+/ 4+)$, murmúrios vesiculares bem diminuídos em hemitórax esquerdo, com tiragens supraclavicular e intercostal, saturando 98\% em máscara com reservatório e frequência respiratória de 60 incursões por minuto.

A equipe médica levantou como hipóteses broncopneumonia e aspiração de corpo estranho, sendo realizada de urgência uma tomografia computadorizada de tórax (Figura 2) cujo resultado foi sugestivo de HDC. Em seguida, o lactente foi submetido à videotoracoscopia, herniorrafia diafragmática e colocação de dreno torácico em selo d'água, o qual foi retirado no segundo dia pós-operatório e iniciada dieta. O paciente recebeu alta 72 horas após o procedimento.

Figura 1 - Radiografia de tórax normal e contrastado evidenciando o conteúdo de vísceras no tórax

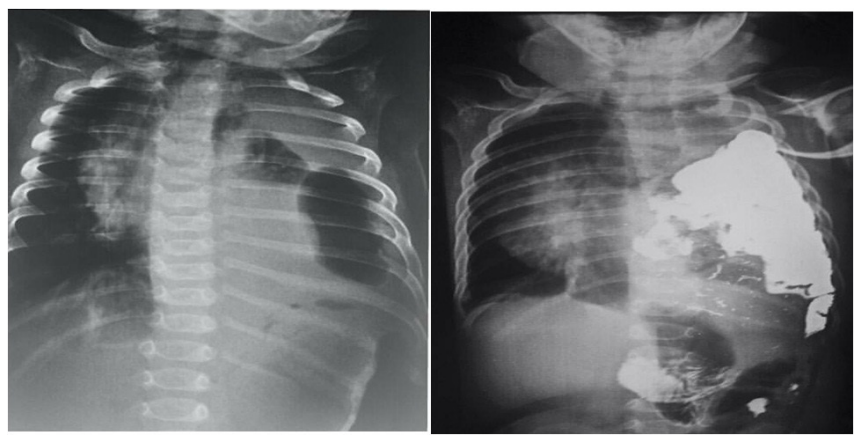

Fonte: Arquivo pessoal (2016).

Figura 2 - Tomografia computadorizada do tórax corte frontal e axial evidenciando herniação e assimetria torácica

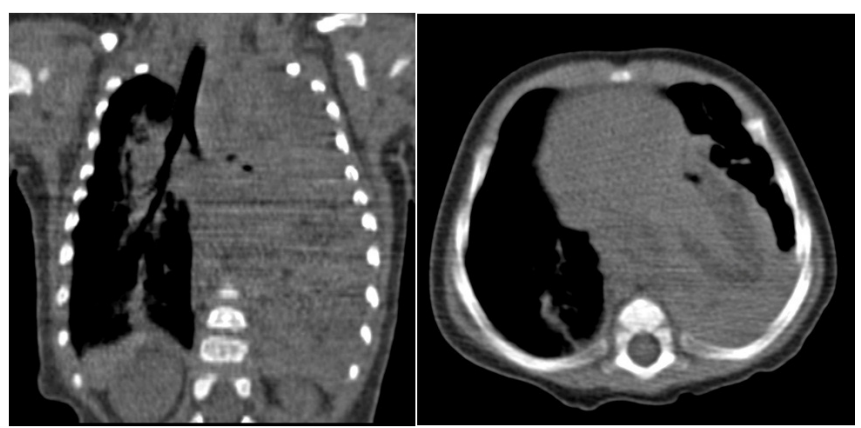

Fonte: Arquivo pessoal (2016).

\section{DISCUSSÃO}

As hérnias diafragmáticas congênitas classificam-se de acordo com a localização do defeito em três tipos: hérnia de Bochdalek, hérnia de Morgagni e hérnia do hiato esofágico. As hérnias de Bochdaleck resultam de um defeito no segmento posterolateral do diafragma e representam 85 a 90\% das HDC. Já as hérnias de Morgagni são resultado de um defeito no segmento anterior, entre 
a origem costal e esternal do diafragma e representam apenas 1 a $2 \%$ das HDC. ${ }^{7}$ Por fim, as hérnias do hiato esofágico resultam do alargamento excessivo do hiato esofágico podendo levar à passagem de estômago para a cavidade torácica. ${ }^{1}$

Atualmente 46 a 97\% dos casos podem ser diagnosticados na ecografia pré-natal antes da $25^{\circ}$ semana de gestação. A ultrassonografia (USG) pode mostrar polidrâmnio, ausência de bolha gástrica abdominal, desvio de mediastino e hidropsia fetal. A USG mostra também a alteração dinâmica da herniação visceral para dentro do tórax na HDC, com as vísceras movendo-se para dentro e para fora do tórax..$^{8,2}$

O diagnóstico pré-natal é de grande importância, uma vez que permite o acompanhamento do feto, além de orientar os pais e familiares quanto às possíveis manifestações e complicações. Permite a intervenção pré-natal quando necessário e conduz a realização do parto em hospital terciário com equipes de neonatologia e cirurgia pediátrica disponíveis para os cuidados imediatos com o recém-nascido (RN) buscando reduzir, dessa forma, a taxa de mortalidade. ${ }^{8}$

O RN com hérnia diafragmática congênita apresenta-se habitualmente com taquipneia, tiragem intercostal e supraclavicular e cianose no período pós-natal imediato, podendo apresentar também abdome escavado, a depender do grau de herniação e assimetria torácica. ${ }^{9} \mathrm{O}$ murmúrio vesicular pode não ser audível no lado acometido, o que, ocasionalmente, leva ao diagnóstico errôneo de pneumotórax hipertensivo, acompanhado por distensão abdominal causada pelo rebaixamento do diafragma. Uma radiografia de tórax e abdome, mostrando as alças intestinais no hemitórax, estabelece o diagnóstico. ${ }^{10}$

Apesar de ter uma pequena incidência (5-10\%), a HDC pode ter manifestação clínica tardia e bem variável, por exemplo esse lactente, que só apresentou sintomas relevantes a partir do $5^{\circ}$ mês de vida. O quadro clínico diversificado pode incluir desde sintomas característicos de obstrução do sistema digestivo, como náuseas, vômitos, dor abdominal e constipação, até sintomas respiratórios variáveis - tosse e faltar de ar intermitente -, bem como dispneia e taquipneia súbitas. ${ }^{11}$ Essa variedade clínica, sobretudo numa criança fora da faixa etária de RN, dificulta o pronto-diagnóstico e aumenta as chances de complicações, como obstrução intestinal, insuficiência cardiorrespiratória e isquemia intestinal. Para o diagnóstico diferencial da apresentação tardia, os exames de imagem tornam-se importantes, tal qual no caso exposto, que teve como hipóteses iniciais a broncopneumonia ou aspiração de corpo estranho, sobretudo a radiografia contrastada e a tomografia de tórax, pois complementam o estudo de uma condição menos comum. ${ }^{12}$ De acordo com os dados de Bagłaj, ${ }^{13}$ a precisão diagnóstica com a radiografia de tórax chega a $49 \%$ e o encontro de alças intestinais com ar, por vezes com níveis hidroaéreos e desvio do mediastino, são sinais típicos de HDC ao raio X. ${ }^{13,14}$

\section{REFERÊNCIAS}

1 Santos E, Ribeiro S. Hérnia diafragmática congênita: artigo de revisão. Acta Obstet Ginecol Port. 2008;2(1):25-33.

2 Wung JT. Hérnia diafragmática congênita. In: Moreira MEL, Lopes JMA, Carvalho M (Orgs.). O recém-nascido de alto risco: teoria e prática do cuidar. Rio de Janeiro: Fiocruz; 2004. p. 509-24

3 Aita JF, Zanolla GR, Barcelos A, Nascimento L, Knebel R, Verney I. Hérnia diafragmática congênita de apresentação tardia: uma possível causa de dificuldade respiratória aguda na criança. J Pediatr. 1999;75(2):135-8.

4 Santos LT, Evangelista TM. Hérnia diafragmática congênita: relato de caso. Revista Ciências em Saúde. 2013;3(1):27-34.

5 Matsudera S, Nakajima M, Takahashi M, Muroi H, Kikuchi M, Shida Y et al. Laparoscopic surgery for a Bochdalek hernia triggered by pregnancy in an adult woman: a case report. Int J Sug Case Rep. 2018;48:10-15.

6 Amim B. O valor da ultrassonografia e da ressonância magnética na avaliação das hérnias diafragmáticas. Radiol Bras. 2010;43(1):66.

7 Sousa C, Lopes T, Coelho A, Marinho S, Bindi R, Castro R. Hérnia diafragmática de Morgagni: um achado radiológico. Nascer e Crescer. 2015;24(Supl II):S30

8 Netto A. Hérnia diafragmática congênita. In: Lopez FA, Burns DAR, Campos Jr. D (Orgs). Tratado de Pediatria. 2. ed. São Paulo: Manole; 2010. p. 2589-93.

9 Ferreira TA, Chagas ISS, Ramos RTT, Souza EL. Malformações torácicas congênitas na infância: experiência de duas décadas. J Bras Pneumol. 2015;41(2):196-9.

10 Gallindo RM, Gonçalves FL, Figueira RL, Sbragia L. Manejo pré-natal da hérnia diafragmática congênita: presente, passado e futuro. Rev Bras Ginecol Obstet. 2015;37(3):140-7.

11 Radovic SV. Late presentation of congenital diaphagmatic hernia: case report. Srp Arh Celok Lek. 2015;143(9-10):604-8.

12 Burgos CM, Frenkner B, Luco M, Harting MT, Lally PA, Lally KP. Prenatally versus postnatally diagnosed congenital diaphragmatic hernia: side, stage and outcome. J Pediatr Surg. 2018;1-5.

13 Bagłaj M. Late-presenting congenital diaphragmatic hernia in children: a clinical spectrum. Pediatr Surg Int. 2004;20(9):658-69.

14 Chao PH, Chuang JH, Lee SY, Huang HC. Late-presenting congenital diaphragmatic hernia in childhood. Acta Paediatr $2011 ; 100(3): 425-8$. 